\title{
A combinatorial formula for rank 2 cluster variables
}

\author{
Kyungyong Lee $\cdot$ Ralf Schiffler
}

Received: 4 August 2011 / Accepted: 10 March 2012 / Published online: 28 March 2012

(C) Springer Science+Business Media, LLC 2012

Abstract Let $r$ be any positive integer, and let $x_{1}, x_{2}$ be indeterminates. We consider the sequence $\left\{x_{n}\right\}$ defined by the recursive relation

$$
x_{n+1}=\left(x_{n}^{r}+1\right) / x_{n-1}
$$

for any integer $n$. Finding a combinatorial expression for $x_{n}$ as a rational function of $x_{1}$ and $x_{2}$ has been an open problem since 2001. We give a direct elementary formula for $x_{n}$ in terms of subpaths of a specific lattice path in the plane. The formula is manifestly positive, providing a new proof of a result by Nakajima and Qin.

Keywords Laurent polynomials · Cluster algebras $\cdot$ Lattice paths

\section{Introduction}

Let $r$ be any positive integer, and $x_{1}, x_{2}$ be indeterminates. We consider the sequence $\left\{x_{n}\right\}$ defined by the recursive relation

$$
x_{n+1}=\left(x_{n}^{r}+1\right) / x_{n-1}
$$

for any integer $n$. These seemingly elementary sequences have turned out to be stubbornly hard to analyze. In the case $r=1$, surprisingly, we obtain a periodic sequence

K. Lee $(\bowtie)$

Department of Mathematics, Wayne State University, Detroit, MI 48202, USA

e-mail:klee@math.wayne.edu

R. Schiffler

Department of Mathematics, University of Connecticut, Storrs, CT 06269, USA

e-mail: schiffler@math.uconn.edu 
of Laurent polynomials of $x_{1}$ and $x_{2}$ :

$$
x_{3}=\frac{x_{2}+1}{x_{1}}, \quad x_{4}=\frac{x_{1}+x_{2}+1}{x_{1} x_{2}}, \quad x_{5}=\frac{x_{1}+1}{x_{2}}, \quad x_{6}=x_{1}, \quad x_{7}=x_{2}, \ldots
$$

In all other cases $r>1$, the sequence is without repetition. It is an important problem in the theory of cluster algebras to understand the sequence for an arbitrary positive integer $r$, because each sequence forms the set of cluster variables of a rank two cluster algebra.

Cluster algebras have been introduced by Fomin and Zelevinsky in [10] in the context of total positivity and canonical bases in Lie theory. Since then cluster algebras have been shown to be related to various fields in mathematics including representation theory of finite dimensional algebras, Teichmüller theory, Poisson geometry, combinatorics, Lie theory, tropical geometry and mathematical physics.

A cluster algebra is a subalgebra of a field of rational functions in $n$ variables $x_{1}, x_{2}, \ldots, x_{n}$, given by specifying a set of generators, the so-called cluster variables. These generators are constructed in a recursive way, starting from the initial variables $x_{1}, x_{2}, \ldots, x_{n}$, by a procedure called mutation, which is determined by the choice of a skew-symmetric $n \times n$ integer matrix $B$. Although each mutation is an elementary operation, it is very difficult to compute cluster variables in general, because of the recursive character of the construction.

Finding explicit computable direct formulas for the cluster variables is one of the main open problems in the theory of cluster algebras and has been studied by many mathematicians. Fomin and Zelevinsky showed in [10] that every cluster variable is a Laurent polynomial in the initial variables $x_{1}, x_{2}, \ldots, x_{n}$, and they conjectured that this Laurent polynomial has positive coefficients. This positivity conjecture has been proved in several special cases; the most general ones being cluster algebras from surfaces, see [17], cluster algebras that have a bipartite seed, see [14, 18] and cluster algebras whose initial seed is acyclic [19].

Direct formulas for the Laurent polynomials have been obtained in several special cases. The most general results are the following:

- a formula involving the Euler-Poincaré characteristic of quiver Grassmannians obtained in [12] using categorification and generalizing results in [5, 6]. While this formula shows a very interesting connection between cluster algebras and geometry, it is of limited computational use, since the Euler-Poincaré characteristics of quiver Grassmannians are hard to compute.

- an elementary combinatorial formula for cluster algebras from surfaces given in [17] building on [20-22].

- a formula for cluster variables corresponding to string modules as a product of $2 \times 2$ matrices obtained in [2], generalizing a result in [3].

In this paper, we consider cluster algebras of rank $n=2$, that is, the integer matrix $B$ is of the form

$$
\left[\begin{array}{cc}
0 & r \\
-r & 0
\end{array}\right]
$$

and the cluster variables form the sequence $\left\{x_{n}\right\}$ given by the recursion above. The rank 2 case is considerably simpler than the general case, but even so, the problem of 
finding an elementary formula for the cluster variables which also shows positivity had not been solved yet. In [7, 8, 16, 23] such formulas are given in the case $r=2$, which is also a special case of the ones considered in $[2,17,22]$. In $[18,19]$ the positivity conjecture was proved for arbitrary $r$ using Euler-Poincaré characteristics, and in [15] an elementary formula is proved but it does not show positivity. We also want to point out that the positivity conjecture has been shown in the case where the $2 \times 2$ matrix $B$ is skew-symmetrizable in [9], again using Euler-Poincaré characteristics.

The main result of this paper is a direct combinatorial formula which computes the cluster variables as a sum of monomials each with coefficient 1. Clearly, this formula shows positivity. Moreover, each monomial is explicitly given, and the sum is parametrized by subpaths of a specific lattice path in the plane.

\section{Main result}

Fix a positive integer $r \geq 2$.

Definition 1 Let $\left\{c_{n}\right\}$ be the sequence defined by the recurrence relation

$$
c_{n}=r c_{n-1}-c_{n-2},
$$

with the initial condition $c_{1}=0, c_{2}=1$. When $r=2, c_{n}=n-1$. When $r>2$, it is easy to see that

$$
\begin{aligned}
c_{n} & =\frac{1}{\sqrt{r^{2}-4}}\left(\frac{r+\sqrt{r^{2}-4}}{2}\right)^{n-1}-\frac{1}{\sqrt{r^{2}-4}}\left(\frac{r-\sqrt{r^{2}-4}}{2}\right)^{n-1} \\
& =\sum_{i \geq 0}(-1)^{i}\left(\begin{array}{c}
n-2-i \\
i
\end{array}\right) r^{n-2-2 i} .
\end{aligned}
$$

For example, for $r=3$, the sequence $c_{n}$ takes the following values:

$$
0,1,3,8,21,55,144, \ldots
$$

In order to state our theorem, we fix an integer $n \geq 4$. Consider a rectangle with vertices $(0,0),\left(0, c_{n-2}\right),\left(c_{n-1}-c_{n-2}, c_{n-2}\right)$ and $\left(c_{n-1}-c_{n-2}, 0\right)$. In what follows, by the diagonal we mean the line segment from $(0,0)$ to $\left(c_{n-1}-c_{n-2}, c_{n-2}\right)$. A Dyck path is a lattice path from $(0,0)$ to $\left(c_{n-1}-c_{n-2}, c_{n-2}\right)$ that proceeds by NORTH or EAST steps and never goes above the diagonal.

Definition 2 A Dyck path below the diagonal is said to be maximal if no subpath of any other Dyck path lies above it. The maximal Dyck path, denoted by $\mathcal{D}_{n}$, consists of $\left(w_{0}, \alpha_{1}, w_{1}, \ldots, \alpha_{c_{n-1}}, w_{c_{n-1}}\right)$, where $w_{0}, \ldots, w_{c_{n-1}}$ are vertices and $\alpha_{1}, \ldots, \alpha_{c_{n-1}}$ are edges, such that $w_{0}=(0,0)$ is the south-west corner of the rectangle, $\alpha_{i}$ connects $w_{i-1}$ and $w_{i}$, and $w_{c_{n-1}}=\left(c_{n-1}-c_{n-2}, c_{n-2}\right)$ is the north-east corner of the rectangle. 
Remark 3 The word obtained from $\mathcal{D}_{n}$ by forgetting the vertices $w_{i}$ and replacing each horizontal edge by the letter $x$ and each vertical edge by the letter $y$ is the Christoffel word of slope $c_{n-2} /\left(c_{n-1}-c_{n-2}\right)$, see for example [4].

Example 4 Let $r=3$ and $n=5$. Then $\mathcal{D}_{5}$ is illustrated as follows:

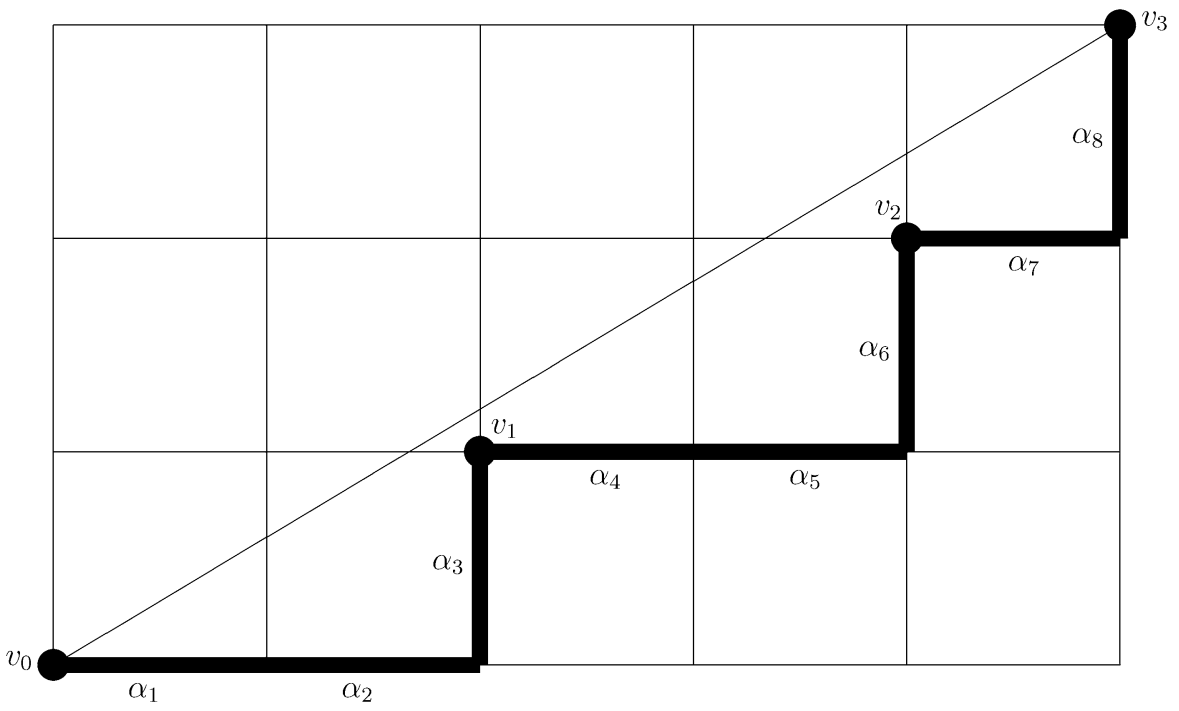

Definition 5 Let $i_{1}<\cdots<i_{c_{n-2}}$ be the sequence of integers such that $\alpha_{i_{j}}$ is vertical for any $1 \leq j \leq c_{n-2}$. Define a sequence $v_{0}, v_{1}, \ldots, v_{c_{n-2}}$ of vertices by $v_{0}=(0,0)$ and $v_{j}=w_{i_{j}}$.

Definition 6 For any $i<j$, let $s_{i, j}$ be the slope of the line through $v_{i}$ and $v_{j}$. Let $s$ be the slope of the diagonal, that is, $s=s_{0, c_{n-2}}$.

Definition 7 For any $0 \leq i<k \leq c_{n-2}$, we define a colored subpath $\alpha(i, k)$ to be the subpath of $\mathcal{D}_{n}$ defined as follows (for illustrations see Example 11).

(1) If $s_{i, t} \leq s$ for all $t$ such that $i<t \leq k$, then let $\alpha(i, k)$ be the subpath from $v_{i}$ to $v_{k}$. Each of these subpaths will be called a BLUE subpath. See Example 11.

(2) If $s_{i, t}>s$ for some $i<t \leq k$, then

(2-a) If the smallest such $t$ is of the form $i+c_{m}-w c_{m-1}$ for some integers $3 \leq m \leq n-2$ and $1 \leq w<r-1$, then let $\alpha(i, k)$ be the subpath from $v_{i}$ to $v_{k}$. Each of these subpaths will be called a GREEN subpath. When $m$ and $w$ are specified, it will be said to be $(m, w)$-green;

(2-b) Otherwise, let $\alpha(i, k)$ be the subpath from the immediate predecessor of $v_{i}$ to $v_{k}$. Each of these subpaths will be called a RED subpath.

Note that every pair $(i, k)$ defines exactly one subpath $\alpha(i, k)$. We denote the set of all these subpaths together with the single edges $\alpha_{i}$ by $\mathcal{P}\left(\mathcal{D}_{n}\right)$, that is,

$$
\mathcal{P}\left(\mathcal{D}_{n}\right)=\left\{\alpha(i, k) \mid 0 \leq i<k \leq c_{n-2}\right\} \cup\left\{\alpha_{1}, \ldots, \alpha_{c_{n-1}}\right\} .
$$


Now we define a set $\mathcal{F}\left(\mathcal{D}_{n}\right)$ of certain sequences of non-overlapping subpaths of $\mathcal{D}_{n}$. This set will parametrize the monomials in our expansion formula.

\section{Definition 8 Let}

$$
\begin{aligned}
\mathcal{F}\left(\mathcal{D}_{n}\right)= & \left\{\left\{\beta_{1}, \ldots, \beta_{t}\right\} \mid t \geq 0, \beta_{j} \in \mathcal{P}\left(\mathcal{D}_{n}\right) \text { for all } 1 \leq j \leq t,\right. \\
& \text { if } j \neq j^{\prime} \text { then } \beta_{j} \text { and } \beta_{j^{\prime}} \text { have no common edge, } \\
& \text { if } \beta_{j}=\alpha(i, k) \text { and } \beta_{j^{\prime}}=\alpha\left(i^{\prime}, k^{\prime}\right) \text { then } i \neq k^{\prime} \text { and } i^{\prime} \neq k, \\
& \text { and if } \beta_{j} \text { is }(m, w) \text {-green then at least one of the }\left(c_{m-1}-w c_{m-2}\right) \\
& \text { preceding edges of } \left.v_{i} \text { is contained in some } \beta_{j^{\prime}}\right\}
\end{aligned}
$$

For any $\beta=\left\{\beta_{1}, \ldots, \beta_{t}\right\}$, let $|\beta|_{2}$ be the total number of edges in $\beta_{1}, \ldots, \beta_{t}$, and $|\beta|_{1}=\sum_{j=1}^{t}\left|\beta_{j}\right|_{1}$, where

$$
\left|\beta_{j}\right|_{1}= \begin{cases}0, & \text { if } \beta_{j}=\alpha_{i} \text { for some } 1 \leq i \leq c_{n-1} \\ k-i, & \text { if } \beta_{j}=\alpha(i, k) \text { for some } 0 \leq i<k \leq c_{n-2}\end{cases}
$$

Theorem 9 Let $r \geq 2$ be a positive integer. Let $x_{1}, x_{2}$ be indeterminates. Define the sequence $\left\{x_{n}\right\}$ by

$$
x_{n+1}=\left(x_{n}^{r}+1\right) / x_{n-1} \quad \text { for any integer } n .
$$

Then for $n \geq 4$,

$$
x_{n}=x_{1}^{-c_{n-1}} x_{2}^{-c_{n-2}} \sum_{\beta \in \mathcal{F}\left(\mathcal{D}_{n}\right)} x_{1}^{r|\beta|_{1}} x_{2}^{r\left(c_{n-1}-|\beta|_{2}\right)}
$$

and

$$
x_{3-n}=x_{2}^{-c_{n-1}} x_{1}^{-c_{n-2}} \sum_{\beta \in \mathcal{F}\left(\mathcal{D}_{n}\right)} x_{2}^{r|\beta|_{1}} x_{1}^{r\left(c_{n-1}-|\beta|_{2}\right)} .
$$

Remark 10 We can rewrite (2.1) as

$$
x_{n}=x_{1}^{-c_{n-1}} x_{2}^{-c_{n-2}} \sum \sum x_{1}^{r|\beta|_{1}} x_{2}^{r\left(c_{n-1}-|\beta|_{2}\right)}
$$

where the first sum is over all sequences

$$
0 \leq i_{1}<k_{1}<i_{2}<k_{2}<\cdots<i_{e}<k_{e} \leq c_{n-2}
$$

and the second sum is over all $\beta \in \mathcal{F}\left(\mathcal{D}_{n}\right)$ whose colored subpaths are precisely the $\alpha\left(i_{j}, k_{j}\right)$ for $1 \leq j \leq e$.

Here $x_{n}(n \in \mathbb{Z})$ are called cluster variables, and the cluster algebra of rank 2 is the $\mathbb{Q}$-subalgebra generated by all cluster variables $x_{n}$ in the field of rational functions in the commutative variables $x_{1}$ and $x_{2}$. Note that $x_{3}$ and $x_{0}$ are directly given by the recurrence. 
Example 11 Let $r=3$ and $n=5$. The illustrations below show the possible configurations for $\beta \in \mathcal{F}\left(\mathcal{D}_{n}\right)$. If the edge $\alpha_{i}$ is marked $-\mathbf{m}$, then $\alpha_{i}$ can occur in $\beta$.

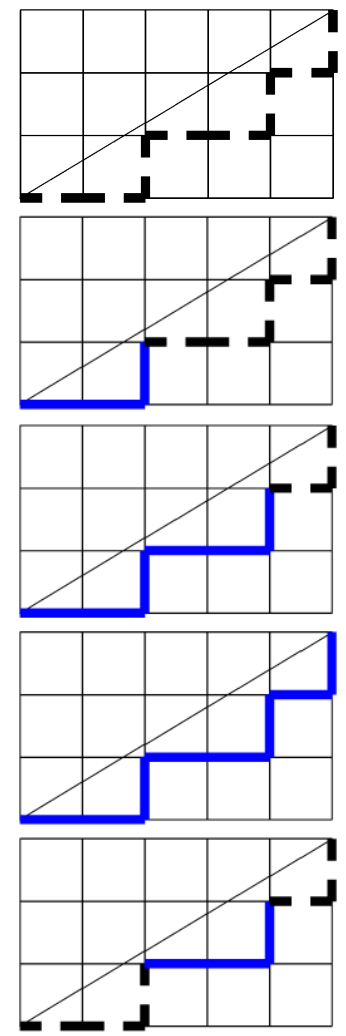

$\sum_{\beta \subset\left\{\alpha_{1}, \ldots, \alpha_{8}\right\}} x_{1}^{r|\beta|_{1}} x_{2}^{r\left(c_{n-1}-|\beta|_{2}\right)}=\left(1+x_{2}^{3}\right)^{8}$

$\sum_{\{\alpha(0,1)\} \subset \beta \subset\{\alpha(0,1)\} \cup\left\{\alpha_{4}, \ldots, \alpha_{8}\right\}} x_{1}^{r|\beta|_{1}} x_{2}^{r\left(c_{n-1}-|\beta|_{2}\right)}=x_{1}^{3}\left(1+x_{2}^{3}\right)^{5}$

$\sum_{\{\alpha(0,2)\} \subset \beta \subset\{\alpha(0,2)\} \cup\left\{\alpha_{7}, \alpha_{8}\right\}} x_{1}^{r|\beta|_{1}} x_{2}^{r\left(c_{n-1}-|\beta|_{2}\right)}=x_{1}^{6}\left(1+x_{2}^{3}\right)^{2}$

$\sum_{\beta=\{\alpha(0,3)\}} x_{1}^{r|\beta|_{1}} x_{2}^{r\left(c_{n-1}-|\beta|_{2}\right)}=x_{1}^{9}$

$\{\alpha(1,2)\} \subset \beta \subset\{\alpha(1,2)\} \cup\left\{\alpha_{1}, \alpha_{2}, \alpha_{3}, \alpha_{7}, \alpha_{8}\right\}$

$$
\sum x_{1}^{r|\beta|_{1}} x_{2}^{r\left(c_{n-1}-|\beta|_{2}\right)}=x_{1}^{3}\left(1+x_{2}^{3}\right)^{5}
$$

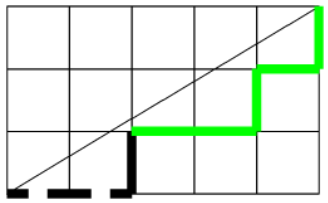

$\{\alpha(1,3)\} \cup\left\{\alpha_{3}\right\} \subset \beta \subset\{\alpha(1,3)\} \cup\left\{\alpha_{1}, \alpha_{2}, \alpha_{3}\right\}$

$$
\sum x_{1}^{r|\beta|_{1}} x_{2}^{r\left(c_{n-1}-|\beta|_{2}\right)}=x_{1}^{6}\left(1+x_{2}^{3}\right)^{2}
$$

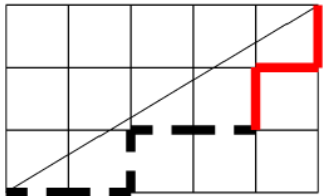

$\sum_{\{\alpha(2,3)\} \subset \beta \subset\{\alpha(2,3)\} \cup\left\{\alpha_{1}, \ldots, \alpha_{5}\right\}} x_{1}^{r|\beta|_{1}} x_{2}^{r\left(c_{n-1}-|\beta|_{2}\right)}=x_{1}^{3}\left(1+x_{2}^{3}\right)^{5}$

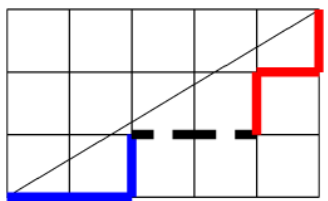

$$
\sum x_{1}^{r|\beta|_{1}} x_{2}^{r\left(c_{n-1}-|\beta|_{2}\right)}=x_{1}^{6}\left(1+x_{2}^{3}\right)^{2}
$$

$\{\alpha(0,1), \alpha(2,3)\} \subset \beta \subset\{\alpha(0,1), \alpha(2,3)\} \cup\left\{\alpha_{4}, \alpha_{5}\right\}$

These configurations are grouped according to Remark 10: in the first picture there are no colored subpath, each of the next four pictures has precisely one blue subpath, 
the sixth picture has a $(3,1)$-green subpath forcing the preceding edge to be included in $\beta$, the seventh picture has a red subpath and the last picture has a blue subpath and a red subpath.

Adding the above eight polynomials together gives

$$
\begin{aligned}
& x_{2}{ }^{24}+8 x_{2}{ }^{21}+3 x_{1}{ }^{3} x_{2}{ }^{15}+28 x_{2}{ }^{18}+15 x_{1}{ }^{3} x_{2}{ }^{12}+56 x_{2}{ }^{15}+3 x_{1}{ }^{6} x_{2}{ }^{6} \\
& +30 x_{1}{ }^{3} x_{2}{ }^{9}+70 x_{2}{ }^{12}+x_{1}{ }^{9}+6 x_{1}{ }^{6} x_{2}{ }^{3}+30 x_{1}{ }^{3} x_{2}{ }^{6}+56 x_{2}{ }^{9}+3 x_{1}{ }^{6} \\
& +15 x_{1}{ }^{3} x_{2}{ }^{3}+28 x_{2}{ }^{6}+3 x_{1}{ }^{3}+8 x_{2}{ }^{3}+1 .
\end{aligned}
$$

Then $x_{5}$ is obtained by dividing (2.3) by $x_{1}^{8} x_{2}^{3}$.

We also obtain the following formula for the $F$-polynomials. Let $g_{\ell}$ be the $g$-vector and let $F_{\ell}$ be the $F$-polynomial of $x_{\ell}$, for all integers $\ell$. Then $g_{3}=$ $(-1, r), g_{0}=(0,-1), F_{3}=y_{1}+1$ and $F_{0}=y_{2}+1$, and all other cases are described in the following result.

\section{Corollary 12 Let $n \geq 4$. Then}

$$
\begin{aligned}
& g_{n}=\left(-c_{n-1}, c_{n}\right), \quad g_{3-n}=\left(-c_{n-2}, c_{n-3}\right), \quad \text { and } \\
& F_{n}=\sum_{\beta \in \mathcal{F}\left(\mathcal{D}_{n}\right)} y_{1}^{|\beta|_{2}} y_{2}^{|\beta|_{1}}, \quad F_{3-n}=\sum_{\beta \in \mathcal{F}\left(\mathcal{D}_{n}\right)} y_{1}^{c_{n-2}-|\beta|_{1}} y_{2}^{c_{n-1}-|\beta|_{2}} .
\end{aligned}
$$

Proof The formulas for the $g$-vectors follow easily from the recursive relations for $g$-vectors given in [11, Proposition 6.6]. To prove the formulas for the $F$-polynomials, we work in the cluster algebra with principal coefficients at the seed

$$
\Sigma=\left(\left(x_{1}, x_{2}\right),\left(y_{1}, y_{2}\right),\left[\begin{array}{cc}
0 & r \\
-r & 0 \\
1 & 0 \\
0 & 1
\end{array}\right]\right)
$$

In this cluster algebra, the Laurent expansion in $\Sigma$ of any cluster variable $x_{\ell}$ is homogeneous with respect to the $\mathbb{Z}^{2}$-grading

$$
\operatorname{deg} x_{1}=(1,0), \quad \operatorname{deg} x_{2}=(0,1), \quad \operatorname{deg} y_{1}=(0, r), \quad \operatorname{deg} y_{2}=(-r, 0),
$$

and, moreover, $\operatorname{deg} x_{\ell}=g_{\ell}$, see [11, Proposition 6.1]. It follows that the expansion formulas with principal coefficients are of the form

$$
x_{n}=x_{1}^{-c_{n-1}} x_{2}^{-c_{n-2}} \sum_{\beta \in \mathcal{F}\left(\mathcal{D}_{n}\right)} x_{1}^{r|\beta|_{1}} x_{2}^{r\left(c_{n-1}-|\beta|_{2}\right)} y_{1}^{a_{1}} y_{2}^{a_{2}}
$$

and

$$
x_{3-n}=x_{2}^{-c_{n-1}} x_{1}^{-c_{n-2}} \sum_{\beta \in \mathcal{F}\left(\mathcal{D}_{n}\right)} x_{2}^{r|\beta|_{1}} x_{1}^{r\left(c_{n-1}-|\beta|_{2}\right)} y_{1}^{b_{1}} y_{2}^{b_{2}},
$$


where $a_{1}, a_{2}, b_{1}$ and $b_{2}$ are integers such that

$$
g_{n}=\operatorname{deg} x_{n}=\left(-c_{n-1}+r|\beta|_{1}-r a_{2},-c_{n-2}+r\left(c_{n-1}-|\beta|_{2}\right)+r a_{1}\right)
$$

and

$$
g_{3-n}=\operatorname{deg} x_{3-n}=\left(-c_{n-2}+r\left(c_{n-1}-|\beta|_{2}\right)-r b_{2},-c_{n-1}+r|\beta|_{1}+r b_{1}\right) .
$$

Now, using the formulas for the $g$-vectors in the corollary and the recurrence $c_{n}=$ $r c_{n-1}-c_{n-2}$, we get $a_{1}=|\beta|_{2}, a_{2}=|\beta|_{1}, b_{1}=c_{n-2}-|\beta|_{1}$ and $b_{2}=c_{n-1}-|\beta|_{2}$. The formulas for the $F$-polynomials now follow by setting $x_{1}=x_{2}=1$ in (2.4) and (2.5).

Theorem 9 also enables us to compute the Euler-Poincaré characteristics of certain quiver Grassmannians. Let $Q_{r}$ be the generalized Kronecker quiver with two vertices 1 and 2, and $r$ arrows from 1 to 2. For $n \geq 3$, let $M(n)$ (resp. $M(3-n)$ ) be the unique (up to an isomorphism) indecomposable representation of dimension vector $\left(c_{n-1}, c_{n-2}\right)$ (resp. $\left.\left(c_{n-2}, c_{n-1}\right)\right)$. Then the indecomposable projective representations are $P(2)=M(0)$ and $P(1)=M(-1)$, and any indecomposable preprojective representation is of the form $M(3-n)$. Similarly, the indecomposable injective representations are $I(1)=M(3)$ and $I(2)=M(4)$, and any indecomposable preinjective representation is of the form $M(n)$. Let $\operatorname{Gr}_{\left(e_{1}, e_{2}\right)}(M(n))\left(\operatorname{resp} \cdot \operatorname{Gr}_{\left(e_{1}, e_{2}\right)}(M(3-n))\right)$ be the variety parametrizing all subrepresentations of $M(n)(\operatorname{resp} . M(3-n))$ of dimension vector $\left(e_{1}, e_{2}\right)$. We use a result of Caldero and Zelevinsky [7, Theorem 3.2 and (3.5)].

Theorem 13 (Caldero and Zelevinsky) Let $n \geq 3$. Then the cluster variable $x_{n}$ is equal to

$$
x_{1}^{-c_{n-1}} x_{2}^{-c_{n-2}} \sum_{e_{1}, e_{2}} \chi\left(\operatorname{Gr}_{\left(e_{1}, e_{2}\right)}(M(n))\right) x_{1}^{r\left(c_{n-2}-e_{2}\right)} x_{2}^{r e_{1}},
$$

and $x_{3-n}$ is equal to

$$
x_{1}^{-c_{n-2}} x_{2}^{-c_{n-1}} \sum_{e_{1}, e_{2}} \chi\left(\operatorname{Gr}_{\left(e_{1}, e_{2}\right)}(M(3-n))\right) x_{1}^{r\left(c_{n-1}-e_{2}\right)} x_{2}^{r e_{1}} .
$$

In the case $n=3$, the representations $M(3)$ and $M(0)$ are simple representation, meaning that they do not have non-trivial subrepresentations. Therefore the EulerPoincaré characteristics of their Grassmannians are non-zero if and only if the dimension vector $\left(e_{1}, e_{2}\right)$ is equal to $(0,0)$ or equal to the dimension of the simple representation itself, in which cases the Euler-Poincaré characteristic is 1 . For $n \geq 4$, we get the following result.

Corollary 14 Let $n \geq 4$. Then for any integers $e_{1}$ and $e_{2}$,

$$
\chi\left(\operatorname{Gr}_{\left(e_{1}, e_{2}\right)}(M(n))\right)=\#\left\{\beta \in \mathcal{F}\left(\mathcal{D}_{n}\right):|\beta|_{1}=c_{n-2}-e_{2},|\beta|_{2}=c_{n-1}-e_{1}\right\}
$$

and

$$
\chi\left(\operatorname{Gr}_{\left(e_{1}, e_{2}\right)}(M(3-n))\right)=\#\left\{\beta \in \mathcal{F}\left(\mathcal{D}_{n}\right):|\beta|_{1}=e_{1},|\beta|_{2}=e_{2}\right\}
$$




\section{Proofs}

Since (2.2) can be easily obtained from (2.1) by interchanging $x_{1}$ and $x_{2}$, we will prove (2.1). In addition to our theoretical proof, our formula is checked by Macaulay 2 for any $r, n$ with $r+n \leq 11$. We need more notation.

Definition 15 For integers $u, n$ with $3 \leq u \leq n-1$, let

$$
\begin{aligned}
\mathcal{T}^{\geq u}\left(\mathcal{D}_{n}\right):=\left\{\left\{\beta_{1}, \ldots, \beta_{t}\right\} \mid\right. & t \geq 1, \beta_{j} \in \mathcal{P}\left(\mathcal{D}_{n}\right) \text { for all } 1 \leq j \leq t, \\
& \text { if } j \neq j^{\prime} \text { then } \beta_{j} \text { and } \beta_{j^{\prime}} \text { have no common edge, } \\
& \text { if } \beta_{j}=\alpha(i, k) \text { and } \beta_{j^{\prime}}=\alpha\left(i^{\prime}, k^{\prime}\right) \text { then } i \neq k^{\prime} \text { and } i^{\prime} \neq k, \\
& \text { and there exist integers } j, w, m, \text { with } m \geq u \text { such that } \\
& \beta_{j} \text { is }(m, w) \text {-green and none of the }\left(c_{m-1}-w c_{m-2}\right) \\
& \text { preceding edges of } \left.v_{i} \text { is contained in any } \beta_{j^{\prime}}\right\} .
\end{aligned}
$$

The definition of $\mathcal{T}^{\geq}\left(\mathcal{D}_{n}\right)$ is similar to the definition of $\mathcal{F}\left(\mathcal{D}_{n}\right)$ with the difference that the elements of $\mathcal{T} \geq u\left(\mathcal{D}_{n}\right)$ violate the condition from green paths which is required for the elements in $\mathcal{F}\left(\mathcal{D}_{n}\right)$.

\section{Definition 16 Let}

$\widetilde{\mathcal{F}}\left(\mathcal{D}_{n}\right)=\left\{\left\{\beta_{1}, \ldots, \beta_{t}\right\} \mid t \geq 0, \beta_{j} \in \mathcal{P}\left(\mathcal{D}_{n}\right)\right.$ for all $1 \leq j \leq t$,

if $j \neq j^{\prime}$ then $\beta_{j}$ and $\beta_{j^{\prime}}$ have no common edge,

$$
\text { and if } \left.\beta_{j}=\alpha(i, k) \text { and } \beta_{j^{\prime}}=\alpha\left(i^{\prime}, k^{\prime}\right) \text { then } i \neq k^{\prime} \text { and } i^{\prime} \neq k\right\} \text {. }
$$

The definition of $\tilde{\mathcal{F}}\left(\mathcal{D}_{n}\right)$ is the same as the definition $\mathcal{F}\left(\mathcal{D}_{n}\right)$ but without the restrictions from green paths. Moreover

$$
\mathcal{F}\left(\mathcal{D}_{n}\right)=\tilde{\mathcal{F}}\left(\mathcal{D}_{n}\right) \backslash \mathcal{T}^{\geq 3}\left(\mathcal{D}_{n}\right) .
$$

Lemma 17 If $m \geq n-1$, then there do not exist $i, w(1 \leq w<r-1)$ such that $\min \left\{t \mid i<t \leq c_{n-2}, s_{i, t}>s\right\}$ is of the form $i+c_{m}-w c_{m-1}$. In particular, for any $n \geq 4$, the set $\mathcal{T}^{\geq n-1}\left(\mathcal{D}_{n}\right)$ is empty.

Proof First note that $c_{m}-w c_{m-1}$ is an increasing sequence; this can be shown by induction using the facts that $w \leq r-2$ and $c_{m}=r c_{m-1}-c_{m-2}$. Thus if $m \geq n-1$ and $\min \left\{t \mid i<t \leq c_{n-2}, s_{i, t}>s\right\}=i+c_{m}-w c_{m-1}$, then $\min \left\{t \mid i<t \leq c_{n-2}\right.$, $\left.s_{i, t}>s\right\} \geq c_{n-1}-w c_{n-2}=c_{n-2}(r-w)-c_{n-3}>c_{n-2}(r-w-1) \geq c_{n-2}$. But this is a contradiction, because $v_{c_{n-2}}$ is the highest vertex in $\mathcal{D}_{n}$.

Let $z_{3}=x_{3}$ and

$$
z_{n}=x_{1}^{-c_{n-1}} x_{2}^{-c_{n-2}} \sum_{\beta \in \widetilde{\mathcal{F}}\left(\mathcal{D}_{n}\right)} x_{1}^{r|\beta|_{1}} x_{2}^{r\left(c_{n-1}-|\beta|_{2}\right)}
$$

for $n \geq 4$. 
Let $K=\mathbb{Q}\left(x_{1}, x_{2}\right)$ be the field of rational functions in the commutative variables $x_{1}$ and $x_{2}$. Let $F$ be the automorphism of $K$, which is defined by

$$
F:\left\{\begin{array}{l}
x_{1} \mapsto x_{2} \\
x_{2} \mapsto \frac{1+x_{2}^{r}}{x_{1}}
\end{array}\right.
$$

Lemma 18 Let $n \geq 3$. Then

$$
z_{n+1}=F\left(z_{n}\right)+x_{1}^{-c_{n}} x_{2}^{-c_{n-1}} \sum_{\beta \in \mathcal{T} \geq 3\left(\mathcal{D}_{n+1}\right) \backslash \mathcal{T} \geq 4\left(\mathcal{D}_{n+1}\right)} x_{1}^{r|\beta|_{1}} x_{2}^{r\left(c_{n}-|\beta|_{2}\right)} .
$$

Lemma 19 Let $u \geq 3$ and $n \geq u+2$. Then

$$
\begin{gathered}
F\left(x_{1}^{-c_{n-1}} x_{2}^{-c_{n-2}} \sum_{\beta \in \mathcal{T} \geq u} \sum_{\left(\mathcal{D}_{n}\right) \backslash \mathcal{T} \geq u+1} x_{1}^{\left.r|\beta|_{1}\right)} x_{2}^{r\left(c_{n-1}-|\beta|_{2}\right)}\right) \\
=x_{1}^{-c_{n}} x_{2}^{-c_{n-1}} \sum_{\beta \in \mathcal{T} \geq u+1} \sum_{\left(\mathcal{D}_{n+1}\right) \backslash \mathcal{T} \geq u+2} x_{1}^{r|\beta|_{1}} x_{2}^{r\left(c_{n}-|\beta|_{2}\right)} .
\end{gathered}
$$

Lemma 20 Let $n \geq 4$. Then

$$
\begin{aligned}
x_{n} & =z_{n}-\sum_{m=5}^{n} F^{n-m}\left(x_{1}^{-c_{m-1}} x_{2}^{-c_{m-2}} \sum_{\beta \in \mathcal{T} \geq 3\left(\mathcal{D}_{m}\right) \backslash \mathcal{T} \geq 4\left(\mathcal{D}_{m}\right)} x_{1}^{r|\beta|_{1}} x_{2}^{r\left(c_{m-1}-|\beta|_{2}\right)}\right) \\
& =x_{1}^{-c_{n-1}} x_{2}^{-c_{n-2}} \sum_{\beta \in \mathcal{F}\left(\mathcal{D}_{n}\right)} x_{1}^{r|\beta|_{1}} x_{2}^{r\left(c_{n-1}-|\beta|_{2}\right)}
\end{aligned}
$$

The proof of Lemma 18 will be independent of those of Lemmas 19 and 20. We prove Lemmas 19 and 20 by the following induction:

$$
\begin{aligned}
& \text { [Lemma } 19 \text { holds true for } n \leq d] \\
& \Longrightarrow[\text { Lemma } 20 \text { holds true for } n \leq d+1] \\
& \Longrightarrow[\text { Lemma } 19 \text { holds true for } n \leq d+1] \\
& \Longrightarrow[\text { Lemma } 20 \text { holds true for } n \leq d+2] \cdots
\end{aligned}
$$

Proof of Lemma 20 We use induction on $n$. It is easy to show that $x_{4}=z_{4}$. Assume that (3.4) holds for $n$. 
Then

$$
\begin{aligned}
& x_{n+1}=F\left(x_{n}\right) \\
& \stackrel{\text { F:homomorphism }}{=} F\left(z_{n}\right) \\
& -\sum_{m=5}^{n} F^{n-m+1}\left(x_{1}^{-c_{m-1}} x_{2}^{-c_{m-2}} \sum_{\beta \in \mathcal{T} \geq 3\left(\mathcal{D}_{m}\right) \backslash \mathcal{T} \geq 4\left(\mathcal{D}_{m}\right)} x_{1}^{r|\beta|_{1}} x_{2}^{r\left(c_{m-1}-|\beta|_{2}\right)}\right) \\
& \stackrel{\text { Lemma }}{=} 18 z_{n+1} \\
& -\sum_{m=5}^{n+1} F^{n-m+1}\left(x_{1}^{-c_{m-1}} x_{2}^{-c_{m-2}} \sum_{\beta \in \mathcal{T} \geq 3\left(\mathcal{D}_{m}\right) \backslash \mathcal{T} \geq 4\left(\mathcal{D}_{m}\right)} x_{1}^{r|\beta|_{1}} x_{2}^{r\left(c_{m-1}-|\beta|_{2}\right)}\right) \\
& \stackrel{\text { Lemma }}{=}{ }^{19} z_{n+1}-\sum_{m=5}^{n+1} x_{1}^{-c_{n}} x_{2}^{-c_{n-1}} \sum_{\beta \in \mathcal{T} \geq n-m+4} x_{\left(\mathcal{D}_{n+1}\right) \backslash \mathcal{T} \geq n-m+5} x_{1}^{r|\beta|_{1}} x_{2}^{r\left(c_{n+1}-|\beta|_{2}\right)} \\
& =z_{n+1}-x_{1}^{-c_{n}} x_{2}^{-c_{n-1}} \sum_{\beta \in \mathcal{T} \geq 3} x_{1}^{r|\beta|_{1}} x_{2}^{r\left(c_{n}-|\beta|_{2}\right)} \\
& \stackrel{\text { Lemma }}{=} z_{n+1}-x_{1}^{-c_{n}} x_{2}^{-c_{n-1}} \sum_{\beta \in \mathcal{T} \geq 3\left(\mathcal{D}_{n+1}\right)} x_{1}^{r|\beta|_{1}} x_{2}^{r\left(c_{n}-|\beta|_{2}\right)} \\
& \stackrel{(3.2)}{=} x_{1}^{-c_{n}} x_{2}^{-c_{n-1}} \sum_{\beta \in \widetilde{\mathcal{F}}\left(\mathcal{D}_{n+1}\right) \backslash \mathcal{T} \geq 3\left(\mathcal{D}_{n+1}\right)} x_{1}^{r|\beta|_{1}} x_{2}^{r\left(c_{n}-|\beta|_{2}\right)} \\
& \stackrel{(3.1)}{=} x_{1}^{-c_{n}} x_{2}^{-c_{n-1}} \sum_{\beta \in \mathcal{F}\left(\mathcal{D}_{n+1}\right)} x_{1}^{r|\beta|_{1}} x_{2}^{r\left(c_{n}-|\beta|_{2}\right)} \text {. }
\end{aligned}
$$

In order to prove Lemma 18, we need the following notation.

Definition 21 The sequence $\left\{b_{i, j}\right\}_{i \in \mathbb{Z}_{\geq 2}, 1 \leq j \leq c_{i}}$ is defined by

$$
b_{i, j}= \begin{cases}r, & \text { if } \alpha_{j} \text { is a horizontal edge of } \mathcal{D}_{i+1} \\ r-1, & \text { if } \alpha_{j} \text { is a vertical edge of } \mathcal{D}_{i+1}\end{cases}
$$

For integers $i \leq j$, we denote the set $\{i, i+1, i+2, \ldots, j\}$ by $[i, j]$. We will always identify $[i, j]$ with the subpath given by $\left(\alpha_{i}, \alpha_{i+1}, \ldots, \alpha_{j}\right)$.

Definition 22 We will need a function $f$ from \{subsets of $\left[1, c_{n-1}\right]$ \} to \{subsets of $\left.\left[1, c_{n}\right]\right\}$. For each subset $V \subset\left[1, c_{n-1}\right]$, we define $f(V)$ as follows.

If $V=\emptyset$ then $f(\emptyset)=\emptyset$. If $V \neq \emptyset$ then we write $V$ as a disjoint union of maximal connected subsets $V=\bigsqcup_{i=1}^{j}\left[e_{i}, e_{i}+\ell_{i}-1\right]$ with $\ell_{i}>0(1 \leq i \leq j)$ and $e_{i}+\ell_{i}<$ 
$e_{i+1}(1 \leq i \leq j-1)$. For each $1 \leq i \leq j$, let

$$
W_{i}=\left[1+\sum_{k=1}^{e_{i}-1} b_{n-1, k}, \sum_{k=1}^{e_{i}+\ell_{i}-1} b_{n-1, k}\right]
$$

and define $f_{i}(V)$ by

$$
f_{i}(V):= \begin{cases}W_{i}, & \text { if the subpath given by } W_{i} \text { is blue or green; } \\ \left\{\sum_{k=1}^{e_{i}-1} b_{n-1, k}\right\} \cup W_{i}, & \text { otherwise. }\end{cases}
$$

Then $f(V)$ is obtained by taking the union of $f_{i}(V)$ 's:

$$
f(V):=\bigcup_{i=1}^{j} f_{i}(V)
$$

\section{Remark 23}

(1) The subpath given by $f_{i}(V)$ is always one of blue, green, or red subpaths, every blue, green, or red subpath can be realized as the image of a maximal connected interval under $f$.

(2) If $V$ is the union of maximally connected subsets $V_{i}$, then the images $f\left(V_{i}\right)$ do not overlap and are disconnected.

(3) Each horizontal edge in $V$ is mapped under $f$ to a subpath consisting of $r-1$ horizontal edges and one vertical edge. Each vertical edge in $V$ is mapped to a subpath consisting of $r-2$ horizontal edges and one vertical edge.

Example 24 Let $r=3$ and $n=5$. Then $f(\{4,5,6\})=\{9,10,11,12,13,14,15,16\}$. As illustrated below, the image of the subpath $\left(\alpha_{4}, \alpha_{5}, \alpha_{6}\right)$ under $f$ is the subpath $\left(\alpha_{9}, \ldots, \alpha_{16}\right)$, which is blue.
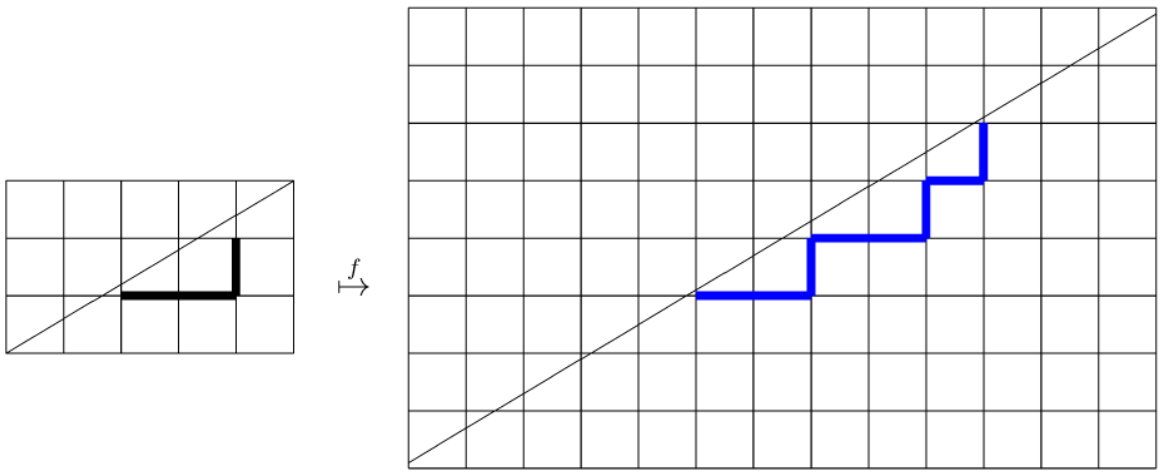
Definition 25 Same notation as in Definition 22. For each $1 \leq i \leq j$, we define $\delta_{\left[e_{i}, e_{i}+\ell_{i}-1\right]}$ by

$$
\begin{cases}1, & \text { if the } w \text {-th edge in the subpath corresponding } \\ & \text { to }\left[e_{i}, e_{i}+\ell_{i}-1\right] \text { is vertical for some } 2 \leq w \leq r-1 \\ 0, & \text { otherwise. }\end{cases}
$$

Let $\delta_{V}:=\sum_{i=1}^{j} \delta_{\left[e_{i}, e_{i}+\ell_{i}-1\right]}$.

Definition 26 (Pull-back) Let $n>1$ be any integer. For any subset $W \subset\left[1, c_{n}\right]$, the pull-back $f^{*}(W)$ by $f$ is defined as follows:

$$
f^{*}(W)=\bigcup_{V: f(V) \subset W} V \subset\left[1, c_{n-1}\right]
$$

The next lemma will be needed to prove Lemma 18.

Lemma 27 Let $V$ be any subset of $\left[1, c_{n-1}\right]$. Then

$$
|f(V)|=r|V|-\left|f^{*}(V)\right|-\delta_{V}
$$

Proof By Remark 23(2), the question is local, so we may assume that $V$ is an interval. First, assume that $V$ is an interval such that the $(r-w)$-th edge in the corresponding subpath, denoted also by $V$, is not vertical for any $1 \leq w \leq r-2$. Observe that $|f(V)|$ is determined by how many horizontal edges and vertical edges are in $V$ and whether the subpath given by $f(V)$ is blue/green or red. Suppose that the subpath given by $f(V)$ is blue or green. Then $|f(V)|=r|V|-$ (the number of vertical edges in $V$ ). Moreover, it follows from Remark 23(3), that if the subpath given by $f(V)$ is blue or green, then (the number of vertical edges in $V)=\left|f^{*}(V)\right|$, because the $(w+1)$ th edge in $V$ is not vertical for any $1 \leq w \leq r-2$.

Suppose that the subpath given by $f(V)$ is red. Then it follows from Remark 23(3) that $|f(V)|=r|V|-$ (the number of vertical edges in $V$ ) +1 . It is not hard to show that (the number of vertical edges in $V)=\left|f^{*}(V)\right|+1$.

If $V$ is an interval such that the $(r-w)$-th edge is vertical for some $1 \leq w \leq r-2$, then it follows, again from Remark 23(3), that $f(V)$ is $(3, w)$-green. Then $|f(V)|=$ $r|V|-$ (the number of vertical edges in $V$ ), and (the number of vertical edges in $V)=\left|f^{*}(V)\right|+1$.

The binomial coefficients we will use are generalized binomial coefficients, i.e., for any (possibly negative) integers $A, B$,

$$
\left(\begin{array}{l}
A \\
B
\end{array}\right):= \begin{cases}\frac{\prod_{i=0}^{B-1}(A-i)}{B !}, & \text { if } B>0 \\
1, & \text { if } B=0 \\
0, & \text { if } B<0\end{cases}
$$




\section{Proof of Lemma 18}

$$
\begin{aligned}
F\left(z_{n}\right) & =x_{2}^{-c_{n-1}} \sum_{\beta \in \widetilde{\mathcal{F}}\left(\mathcal{D}_{n}\right)} x_{2}^{r|\beta|_{1}}\left(\frac{x_{2}^{r}+1}{x_{1}}\right)^{r\left(c_{n-1}-|\beta|_{2}\right)-c_{n-2}} \\
& =x_{1}^{-c_{n}} x_{2}^{-c_{n-1}} \sum_{\beta \in \widetilde{\mathcal{F}}\left(\mathcal{D}_{n}\right)} x_{1}^{r|\beta|_{2}} x_{2}^{r|\beta|_{1}}\left(x_{2}^{r}+1\right)^{c_{n}-r|\beta|_{2}} \\
& =x_{1}^{-c_{n}} x_{2}^{-c_{n-1}} \sum_{\beta \in \widetilde{\mathcal{F}}\left(\mathcal{D}_{n}\right)} \sum_{\theta \in \mathbb{Z}}\left(\begin{array}{c}
c_{n}-r|\beta|_{2} \\
\theta
\end{array}\right) x_{1}^{r|\beta|_{2}} x_{2}^{r|\beta|_{1}} x_{2}^{r \theta} \\
& =x_{1}^{-c_{n}} x_{2}^{-c_{n-1}} \sum_{V \subset\left[1, c_{n-1}\right]} \sum_{\beta: \cup \beta_{i}=V} \sum_{\theta \in \mathbb{Z}}\left(\begin{array}{c}
c_{n}-r|V| \\
\theta
\end{array}\right) x_{1}^{r|V|_{1}} x_{2}^{r|\beta|_{1}} x_{2}^{r \theta} .
\end{aligned}
$$

For a given $V \subset\left[1, c_{n-1}\right]$, consider any subset, say $U$, of $f^{*}(V)$. Then the image of each maximal connected subset of $U$ under $f$ is a blue, green, or red subpath. So $U$ uniquely determines $\beta$, namely,

$\beta=\{f($ all maximal connected subsets of $U)\} \cup\{$ all individual edges in $V \backslash f(U)\}$.

Conversely, $\beta$ with $\bigcup \beta_{i}=V$ uniquely determines a subset $U$ of $f^{*}(V)$, that is,

$$
U=f^{-1} \text { (all blue, green, or red subpaths in } \beta \text { ), }
$$

because $f^{-1}$ (a blue, green, or red subpath) is well-defined and no subpath of $V \backslash$ $f\left(f^{*}(V)\right)$ can be a blue, green, or red subpath.

By Remark 23(3), we have $|\beta|_{1}=|U|$, and therefore $(\dagger \dagger)$ becomes

$F\left(z_{n}\right)$

$$
\begin{aligned}
& =x_{1}^{-c_{n}} x_{2}^{-c_{n-1}} \sum_{V \subset\left[1, c_{n-1}\right]} \sum_{U \subset f^{*}(V)} \sum_{\theta \in \mathbb{Z}}\left(\begin{array}{c}
c_{n}-r|V| \\
\theta
\end{array}\right) x_{1}^{r|V|} x_{2}^{r|U|} x_{2}^{r \theta} \\
& =x_{1}^{-c_{n}} x_{2}^{-c_{n-1}} \sum_{V \subset\left[1, c_{n-1}\right]} \sum_{\rho \in \mathbb{Z}} \sum_{\theta \in \mathbb{Z}}\left(\begin{array}{c}
c_{n}-r|V| \\
\theta
\end{array}\right)\left(\begin{array}{c}
\left|f^{*}(V)\right| \\
\rho
\end{array}\right) x_{1}^{r|V|} x_{2}^{r(\theta+\rho)} \\
& \stackrel{(* *)}{=} x_{1}^{-c_{n}} x_{2}^{-c_{n-1}} \sum_{V \subset\left[1, c_{n-1}\right]} \sum_{\gamma \in \mathbb{Z}}\left(\begin{array}{c}
c_{n}-r|V|+\left|f^{*}(V)\right| \\
\gamma
\end{array}\right) x_{1}^{r|V|} x_{2}^{r \gamma} \\
& =x_{1}^{-c_{n}} x_{2}^{-c_{n-1}} \sum_{V \subset\left[1, c_{n-1}\right]} \sum_{\gamma \in \mathbb{Z}}\left(\begin{array}{c}
c_{n}-r|V|+\left|f^{*}(V)\right| \\
c_{n}-r|V|+\left|f^{*}(V)\right|-\gamma
\end{array}\right) x_{1}^{r|V|} x_{2}^{r\left(c_{n}-r|V|+\left|f^{*}(V)\right|-\gamma\right)} \\
& \sum_{(\text {Lemma } 27)}^{=} x_{1}^{-c_{n}} x_{2}^{-c_{n-1}} \sum_{V \subset\left[1, c_{n-1}\right]}\left(\begin{array}{c}
c_{n}-|f(V)|-\delta_{V} \\
c_{n}-|f(V)|-\delta_{V}-\gamma
\end{array}\right) x_{1}^{r|V|} x_{2}^{r\left(c_{n}-|f(V)|-\delta_{V}-\gamma\right)}
\end{aligned}
$$




$$
\begin{aligned}
& =x_{1}^{-c_{n}} x_{2}^{-c_{n-1}} \sum_{V \subset\left[1, c_{n-1}\right]} \sum_{h \in \mathbb{Z}}\left(\begin{array}{c}
c_{n}-|f(V)|-\delta_{V} \\
c_{n}-|f(V)|-h
\end{array}\right) x_{1}^{r|V|} x_{2}^{r\left(c_{n}-|f(V)|-h\right)} \\
& =x_{1}^{-c_{n}} x_{2}^{-c_{n-1}} \sum_{V \subset\left[1, c_{n-1}\right]} \sum_{h \in \mathbb{Z}}\left(\begin{array}{c}
c_{n}-|f(V)|-\delta_{V} \\
h-\delta_{V}
\end{array}\right) x_{1}^{r|V|} x_{2}^{r\left(c_{n}-|f(V)|-h\right)},
\end{aligned}
$$

where $(* *)$ follows from the $\mathrm{Chu}-$ Vandermonde identity (for instance, see [1, pp. 59-60]).

Next we analyze $z_{n+1}$. If $\beta \in \widetilde{\mathcal{F}}\left(\mathcal{D}_{n+1}\right)$, then there exist

$$
\left\{\begin{array}{l}
e \in \mathbb{Z}_{\geq 0}, \\
0 \leq i_{1}<k_{1}<i_{2}<k_{2}<\cdots<i_{e}<k_{e} \leq c_{n-1}, \\
h \in \mathbb{Z}_{\geq 0}, \text { and } \\
j_{1}<\cdots<j_{h} \in\left[1, c_{n}\right] \backslash f\left(\bigsqcup_{\ell=1}^{e}\left[1+i_{\ell}, k_{\ell}\right]\right)
\end{array}\right.
$$

such that

$$
\beta=\left\{\alpha\left(i_{1}, k_{1}\right), \ldots, \alpha\left(i_{e}, k_{e}\right)\right\} \cup\left\{\alpha_{j_{1}}, \ldots, \alpha_{j_{h}}\right\} .
$$

For a given $\beta \in \widetilde{\mathcal{F}}\left(\mathcal{D}_{n+1}\right)$, this expression is unique. Conversely (3.6) uniquely determines an element $\beta$ in $\widetilde{\mathcal{F}}\left(\mathcal{D}_{n+1}\right)$. Note that $|\beta|_{1}=\sum_{\ell=1}^{e}\left(k_{\ell}-i_{\ell}\right)$ and $|\beta|_{2}=$ $\left|f\left(\bigsqcup_{\ell=1}^{e}\left[1+i_{\ell}, k_{\ell}\right]\right)\right|+h$.

So we have

$$
\begin{aligned}
& z_{n+1}=x_{1}^{-c_{n}} x_{2}^{-c_{n-1}} \sum_{\beta \in \mathcal{\mathcal { F }}\left(\mathcal{D}_{n+1}\right)} x_{1}^{r|\beta|_{1}} x_{2}^{r\left(c_{n}-|\beta|_{2}\right)} \\
& =x_{1}^{-c_{n}} x_{2}^{-c_{n-1}} \sum_{e \in \mathbb{Z}_{\geq 0}} \sum_{1 \leq i_{1}<\cdots<k_{e} \leq c_{n-1}} \sum_{h \in \mathbb{Z}_{\geq 0}} \\
& \sum x_{1}^{r \sum_{l}\left(k_{\ell}-i_{\ell}\right)} x_{2}^{r\left(c_{n}-\left|f\left(\bigsqcup_{\ell}\left[1+i_{\ell}, k_{\ell}\right]\right)\right|-h\right)} \\
& j_{1}<\cdots<j_{h} \in\left[1, c_{n}\right] \backslash f\left(\bigsqcup_{\ell}\left[1+i_{\ell}, k_{\ell}\right]\right) \\
& =x_{1}^{-c_{n}} x_{2}^{-c_{n-1}} \sum_{V \subset\left[1, c_{n-1}\right]} \sum_{h \in \mathbb{Z}_{\geq 0}} \sum_{j_{1}<\cdots<j_{h} \in\left[1, c_{n}\right] \backslash f(V)} x_{1}^{r|V|} x_{2}^{r\left(c_{n}-|f(V)|-h\right)} \\
& =x_{1}^{-c_{n}} x_{2}^{-c_{n-1}} \sum_{V \subset\left[1, c_{n-1}\right]} \sum_{h \in \mathbb{Z}}\left(\begin{array}{c}
c_{n}-|f(V)| \\
h
\end{array}\right) x_{1}^{r|V|} x_{2}^{r\left(c_{n}-|f(V)|-h\right)} \\
& \stackrel{\text { by (†) }}{=} F\left(z_{n}\right)+x_{1}^{-c_{n}} x_{2}^{-c_{n-1}} \sum_{V \subset\left[1, c_{n-1}\right]} \sum_{h \in \mathbb{Z}}\left(\left(\begin{array}{c}
c_{n}-|f(V)| \\
h
\end{array}\right)\right. \\
& \left.-\left(\begin{array}{c}
c_{n}-|f(V)|-\delta_{V} \\
h-\delta_{V}
\end{array}\right)\right) x_{1}^{r|V|} x_{2}^{r\left(c_{n}-|f(V)|-h\right)} \\
& =F\left(z_{n}\right)+x_{1}^{-c_{n}} x_{2}^{-c_{n-1}} \sum_{\beta \in \mathcal{T} \geq 3\left(\mathcal{D}_{n+1}\right) \backslash \mathcal{T} \geq 4\left(\mathcal{D}_{n+1}\right)} x_{1}^{r|\beta|_{1}} x_{2}^{r\left(c_{n}-|\beta|_{2}\right)},
\end{aligned}
$$

where the last equality is a consequence of the next lemma. 
Lemma 28 Let $V$ be any subset of $\left[1, c_{n-1}\right]$ and $h$ be any integer. Then the number of elements $\beta$ in $\widetilde{\mathcal{F}}\left(\mathcal{D}_{n+1}\right)$ satisfying

$$
\left\{\begin{array}{l}
f(V) \text { is the union of all blue, green, and red subpaths in } \beta, \\
|\beta|_{2}=|f(V)|+h, \text { and } \\
\beta \in \mathcal{T}^{\geq 3}\left(\mathcal{D}_{n+1}\right) \backslash \mathcal{T}^{\geq 4}\left(\mathcal{D}_{n+1}\right)
\end{array}\right.
$$

is equal to

$$
\left(\begin{array}{c}
c_{n}-|f(V)| \\
h
\end{array}\right)-\left(\begin{array}{c}
c_{n}-|f(V)|-\delta_{V} \\
h-\delta_{V}
\end{array}\right) .
$$

Proof Remembering the definition of $(m, w)$-green subpaths in Definition 7(2-a), especially for $m=3$, the following statement is a consequence of Remark 23(3). For an interval $[i, k] \subset\left[1, c_{n-1}\right]$, the subpath in $\mathcal{D}_{n}$ corresponding to $[i+1, i+r-1] \cap$ $[i, k]$ contains a vertical edge if and only if the slope in $\mathcal{D}_{n+1}$ between the point $v_{i}$ and the point corresponding to the upper endpoint of the vertical edge is as large as possible, more precisely,

the subpath in $\mathcal{D}_{n+1}$ corresponding to $f([i, k])$ is

$$
(3, w) \text {-green for some } w \in[1, r-2] \text {. }
$$

If no maximal connected interval of $V$ satisfies (3.9), then $\delta_{V}=0$ and there is no $\beta$ satisfying (3.7), so the statement holds true. If only one maximal connected interval, say $[i, k]$, of $V$ satisfies (3.9), then $\delta_{V}=1$. On the other hand, since

$$
\beta \in \mathcal{T}^{\geq 3}\left(\mathcal{D}_{n+1}\right) \backslash \mathcal{T}^{\geq 4}\left(\mathcal{D}_{n+1}\right),
$$

none of the $\left(c_{2}-w c_{1}\right)$ preceding edge(s) of $v_{i}$ is contained in any element $\beta_{j^{\prime}}$ of $\beta$. As $c_{2}-w c_{1}=1$, the number of $\beta$ satisfying (3.7) is obtained by subtracting the number of sequences $j_{1}<\cdots<j_{h} \in\left[1, c_{n}\right] \backslash f(V)$ with $(\min f([i, k])-1) \in$ $\left\{j_{1}, \ldots, j_{h}\right\}$ from the number of sequences $j_{1}<\cdots<j_{h} \in\left[1, c_{n}\right] \backslash f(V)$, which is $\left(\begin{array}{c}c_{n}-|f(V)| \\ h\end{array}\right)-\left(\begin{array}{c}c_{n}-|f(V)|-1 \\ h-1\end{array}\right)$.

Similarly one can verify the statement in the case that more than one maximal connected interval of $V$ satisfies (3.9).

It remains to prove Lemma 19.

Sketch of Proof of Lemma 19 Here we will deal only with the case of $n=u+2$. The case of $n>u+2$ makes use of the same argument. As we use the induction (3.5), we can assume that

$$
x_{i}=x_{1}^{-c_{i-1}} x_{2}^{-c_{i-2}} \sum_{\beta \in \mathcal{F}\left(\mathcal{D}_{i}\right)} x_{1}^{r|\beta|_{1}} x_{2}^{r\left(c_{i-1}-|\beta|_{2}\right)}
$$

for $i \leq n$.

For any $w \in[1, r-2]$, it is easy to show that the lattice point $\left(w\left(c_{n-2}-\right.\right.$ $\left.\left.c_{n-3}\right), w c_{n-3}\right)$ is below the diagonal from $(0,0)$ to $\left(c_{n-1}-c_{n-2}, c_{n-2}\right)$ and that the 
points $\left(w\left(c_{n-2}-c_{n-3}\right), 1+w c_{n-3}\right)$ and $\left(w\left(c_{n-2}-c_{n-3}\right)-1, w c_{n-3}\right)$ are above the diagonal. So $\left(w\left(c_{n-2}-c_{n-3}\right), w c_{n-3}\right)$ is one of the vertices $v_{i}$ on $\mathcal{D}_{n}$. Actually $v_{w c_{n-3}}=\left(w\left(c_{n-2}-c_{n-3}\right), w c_{n-3}\right)$. Since $u=n-2$ and $\alpha\left(w c_{n-3}, c_{n-2}\right)$ is the only $(n-2, w)$-green subpath in $\left\{\alpha(i, k) \mid 0 \leq i<k \leq c_{n-2}\right\}$, every $\beta \in$ $\mathcal{T}^{\geq u}\left(\mathcal{D}_{n}\right) \backslash \mathcal{T}^{\geq u+1}\left(\mathcal{D}_{n}\right)$ must contain the green subpath from $v_{w c_{n-3}}$ to $v_{c_{n-2}}$. Then none of the $c_{n-3}-w c_{n-4}$ preceding edges of $v_{w c_{n-3}}$ is contained in any element $\beta_{j^{\prime}}$ of $\beta$. The green subpath from $v_{w c_{n-3}}$ to $v_{c_{n-2}}$ corresponds to the interval $\left[w c_{n-2}+1, c_{n-1}\right] \subset\left[1, c_{n-1}\right]$. The $c_{n-3}-w c_{n-4}$ preceding edges of $v_{w c_{n-3}}$ are $\alpha_{(r w-1) c_{n-3}+1}, \ldots, \alpha_{w c_{n-2}}$.

Thus we have

$$
\begin{gathered}
\left.x_{1}^{-c_{n-1}} x_{2}^{-c_{n-2}} \sum_{\beta \in \mathcal{T} \geq u\left(\mathcal{D}_{n}\right) \backslash \mathcal{T} \geq u+1} \mathcal{D}_{n}\right) \\
x_{1}^{r|\beta|_{1}} x_{2}^{r\left(c_{n-1}-|\beta|_{2}\right)} \\
\sum_{w=1}^{r-2} x_{1}^{-c_{n-1}} x_{2}^{-c_{n-2}} \sum_{V \subset\left[1,(r w-1) c_{n-3}\right]} \sum_{\beta: \bigcup \beta_{i}=V \cup\left[w c_{n-2}+1, c_{n-1}\right], \beta \ni \alpha\left(w c_{n-3}, c_{n-2}\right)} x_{1}^{r|\beta|_{1}} x_{2}^{r\left(c_{n-1}-|\beta|_{2}\right)} .
\end{gathered}
$$

We observe that the subpath corresponding to $\left[1,(r w-1) c_{n-3}\right]$ consists of $(w-1)$ copies of $\mathcal{D}_{n-1},(r-1)$ copies of $\mathcal{D}_{n-2}$, and $(w-1)$ copies of $\mathcal{D}_{n-3}$. Let $v_{j_{0}}=(0,0)$ and $v_{j_{i}}$ be the end point of each of these copies, i.e.,

$$
\begin{aligned}
& v_{j_{i}}=v_{i c_{n-3}} \quad \text { for } 1 \leq i \leq w-1, \\
& v_{j_{w-1+i}}=v_{(w-1) c_{n-3}+i c_{n-4}} \quad \text { for } 1 \leq i \leq r-1, \\
& v_{j_{w+r-2+i}}=v_{(w-1) c_{n-3}+(r-1) c_{n-4}+i c_{n-5}} \text { for } 1 \leq i \leq w-1 .
\end{aligned}
$$

If a $\left(m, w^{\prime}\right)$-green (resp. blue or red) subpath, say $\alpha(i, k)$, in $\left[1,(r w-1) c_{n-3}\right]$ passes through $v_{j_{e}}, v_{j_{e+1}}, \ldots, v_{j_{e+\ell}}$, then $\alpha(i, k)$ can be naturally decomposed into $\alpha\left(i, j_{e}\right)$, $\alpha\left(j_{e}, j_{e+1}\right), \ldots, \alpha\left(j_{e+\ell}, k\right)$. It is not hard to show that $\alpha\left(i, j_{e}\right)$ is also $\left(m, w^{\prime}\right)$-green (resp. blue or red) and that $\alpha\left(j_{e}, j_{e+1}\right), \ldots, \alpha\left(j_{e+\ell}, k\right)$ are all blue.

Hence

$$
\begin{aligned}
(*)= & \sum_{w=1}^{r-2} x_{1}^{-c_{n-1}} x_{2}^{-c_{n-2}}\left(\sum_{\beta \in \mathcal{F}\left(\mathcal{D}_{n-1}\right)} x_{1}^{r|\beta|_{1}} x_{2}^{r\left(c_{n-2}-|\beta|_{2}\right)}\right)^{w-1} \\
& \times\left(\sum_{\beta \in \mathcal{F}\left(\mathcal{D}_{n-2}\right)} x_{1}^{r|\beta|_{1}} x_{2}^{r\left(c_{n-3}-|\beta|_{2}\right)}\right)^{r-1} \\
& \times\left(\sum_{\beta \in \mathcal{F}\left(\mathcal{D}_{n-3}\right)} x_{1}^{r|\beta|_{1}} x_{2}^{r\left(c_{n-4}-|\beta|_{2}\right)}\right)^{w-1} x_{1}^{r\left(c_{n-2}-w c_{n-3}\right)} x_{2}^{r\left(c_{n-3}-w c_{n-4}\right)} \\
= & \sum_{w=1}^{r-2} x_{1}^{-c_{n-1}} x_{2}^{-c_{n-2}}\left(x_{n-1} x_{1}^{c_{n-2}} x_{2}^{c_{n-3}}\right)^{w-1}\left(x_{n-2} x_{1}^{c_{n-3}} x_{2}^{c_{n-4}}\right)^{r-1} \\
& \times\left(x_{n-3} x_{1}^{c_{n-4}} x_{2}^{c_{n-5}}\right)^{w-1} x_{1}^{r\left(c_{n-2}-w c_{n-3}\right)} x_{2}^{r\left(c_{n-3}-w c_{n-4}\right)}
\end{aligned}
$$




$$
=\sum_{w=1}^{r-2}\left(x_{n-1}\right)^{w-1}\left(x_{n-2}\right)^{r-1}\left(x_{n-3}\right)^{w-1} .
$$

For the same reason, we get

$$
\begin{gathered}
x_{1}^{-c_{n}} x_{2}^{-c_{n-1}} \sum_{\beta \in \mathcal{T} \geq u+1} \sum_{\left(\mathcal{D}_{n+1}\right) \backslash \mathcal{T} \geq u+2} x_{1}^{r|\beta|_{1}} x_{2}^{r\left(c_{n}-|\beta|_{2}\right)} \\
=\sum_{w=1}^{r-2}\left(x_{n}\right)^{w-1}\left(x_{n-1}\right)^{r-1}\left(x_{n-2}\right)^{w-1}
\end{gathered}
$$

Therefore, we have

$$
\begin{aligned}
& F\left(x_{1}^{-c_{n-1}} x_{2}^{-c_{n-2}} \sum_{\beta \in \mathcal{T} \geq u\left(\mathcal{D}_{n}\right) \backslash \mathcal{T} \geq u+1} x_{\left.\mathcal{D}_{n}\right)}^{r|\beta|_{1}} x_{2}^{r\left(c_{n-1}-|\beta|_{2}\right)}\right) \\
& =F\left(\sum_{w=1}^{r-2}\left(x_{n-1}\right)^{w-1}\left(x_{n-2}\right)^{r-1}\left(x_{n-3}\right)^{w-1}\right) \\
& =\sum_{w=1}^{r-2}\left(x_{n}\right)^{w-1}\left(x_{n-1}\right)^{r-1}\left(x_{n-2}\right)^{w-1} \\
& =x_{1}^{-c_{n}} x_{2}^{-c_{n-1}} \sum_{\beta \in \mathcal{T} \geq u+1} x_{1}^{r|\beta|_{1}} x_{2}^{r\left(c_{n}-|\beta|_{2}\right) \backslash \mathcal{T} \geq u+2} .
\end{aligned}
$$

Acknowledgements Research of K.L. is partially supported by NSF grant DMS 0901367. Research of R.S. is partially supported by NSF grant DMS 1001637. The authors are grateful to Andrei Zelevinsky and Hugh Thomas for valuable suggestions. The first author would like to thank Philippe Di Francesco, Sergey Fomin, Rob Lazarsfeld, and Gregg Musiker for encouraging him to work on this project. The computational part of our research was aided by the commutative algebra package Macaulay 2 [13]. The authors thank the referee for helpful comments.

\section{References}

1. Askey, R.: Orthogonal Polynomials and Special Functions. Society for Industrial and Applied Mathematics, Philadelphia (1975). vii+110 pp

2. Assem, I., Dupont, G., Schiffler, R., Smith, D.: Friezes, strings and cluster variables. Glasg. Math. J. 54(1), 27-60 (2012)

3. Assem, I., Reutenauer, C., Smith, D.: Friezes. Adv. Math. 225(6), 3134-3165 (2010)

4. Berstel, J., Lauve, A., Reutenauer, C., Saliola, F.: Combinatorics on Words. Christoffel Words and Repetitions in Words. CRM Monograph Series, vol. 27. American Mathematical Society, Providence (2009)

5. Caldero, P., Chapoton, F.: Cluster algebras as Hall algebras of quiver representations. Comment. Math. Helv. 81(3), 595-616 (2006)

6. Caldero, P., Keller, B.: From triangulated categories to cluster algebras. Invent. Math. 172(1), 169-211 (2008)

7. Caldero, P., Zelevinsky, A.: Laurent expansions in cluster algebras via quiver representations. Mosc. Math. J. 6(3), 411-429 (2006). MR2274858 (2008j:16045) 
8. Di Francesco, P., Kedem, R.: Discrete non-commutative integrability: proof of a conjecture by M. Kontsevich. Intern. Math. Res. Notes (2010). doi:10.1093/imrn/rnq024

9. Dupont, G.: Positivity in coefficient-free rank two cluster algebras. Electron. J. Comb. 16(1), MR2529807 (2009)

10. Fomin, S., Zelevinsky, A.: Cluster algebras I: Foundations. J. Am. Math. Soc. 15(2), 497-529 (2002). MR1887642 (2003f:16050)

11. Fomin, S., Zelevinsky, A.: Cluster algebras IV: Coefficients. Comput. Math. 143, 112-164 (2007)

12. Fu, C., Keller, B.: On cluster algebras with coefficients and 2-Calabi-Yau categories. Trans. Am. Math. Soc. 362(2), 859-895 (2010)

13. Grayson, D., Stillman, M.: Macaulay2, a software system for research in algebraic geometry. Available at http://www.math.uiuc.edu/Macaulay2/

14. Hernandez, D., Leclerc, B.: Cluster algebras and quantum affine algebras. Duke Math. J. 154(2), 265-341 (2010)

15. Lee, K.: On cluster variables of rank two acyclic cluster algebras. To appear in Ann. Comb.

16. Musiker, G., Propp, J.: Combinatorial interpretations for rank-two cluster algebras of affine type. Electron. J. Comb. 14, MR2285819 (2006). (2008j:05374)

17. Musiker, G., Schiffler, R., Williams, L.: Positivity for cluster algebras from surfaces. Adv. Math. 227(6), 2241-2308 (2011)

18. Nakajima, H.: Quiver varieties and cluster algebras. Kyoto J. Math. 51(1), 71-126 (2011)

19. Qin, F.: Quantum cluster variables via Serre polynomials, arXiv:1004.4171. To appear in J. Reine Angewandte Math. (Crelle's Journal)

20. Schiffler, R.: On cluster algebras arising from unpunctured surfaces. II. Adv. Math. 223(6), 18851923 (2010)

21. Schiffler, R.: A cluster expansion formula (an case). Electron. J. Comb. 15(1) 64 (2008). Research paper, 9 pp.

22. Schiffler, R., Thomas, H.: On cluster algebras arising from unpunctured surfaces. Int. Math. Res. Not. 2009(17), 3160-3189 (2009)

23. Sherman, P., Zelevinsky, A.: Positivity and canonical bases in rank 2 cluster algebras of finite and affine types. Mosc. Math. J. 4(4), 947-974 (2004). MR2124174 (2006c:16052) 